\title{
One-Loop Helicity Amplitudes for Parton Level Virtual Compton Scattering
}

\author{
H.W. Huang ${ }^{a}$ and T. Morii ${ }^{b}$ \\ a Department of Physics, University of Colorado, Boulder, CO 80309-0390, USA \\ b Faculty of Human Development, Kobe University, Nada, Kobe 657-8501, Japan
}

\begin{abstract}
We calculate the one-loop QCD virtual corrections to all helicity amplitudes for parton level virtual Compton scattering processes. We include the amplitudes both on quark target process $\gamma^{*} q \rightarrow \gamma q$ and on gluon target process $\gamma^{*} g \rightarrow \gamma g$. The infrared pole structure of the amplitudes is in agreement with the prediction of Catani's general formalism for the singularities of one-loop amplitudes, while expressions for the finite remainder are given in terms of logarithms and dilogarithms that are real in the physical region.
\end{abstract}




\section{Introduction}

Photons, being real or virtual, are known to be clean probes of the internal structure of the nucleon. One of the important processes of photon is Compton scattering, which refers to elastic scattering of a photon off a charged object. The Compton reaction in different kinematical situations can provide different physical information about the behavior of quarks and gluons in the nucleon. There are two complementary kinematical regions, which are deeply virtual region and wide-angle region. The region of deeply virtual Compton scattering (DVCS) is characterized by small momentum transfer from the initial to final nucleon and a large photon virtuality, while in the region of wide-angle Compton scattering (WACS) the situation is reversed.

The theoretical development on DVCS has revealed that in the Bjorken limit, this process is dominated by the simple handbag mechanism in which a quark (an antiquark) in the initial nucleon absorbs the virtual photon, immediately radiates a real photon, and falls back to form the recoiled nucleon [1, 2, 3. It has been proved that DVCS amplitude can be factorized into the finite perturbative parts and the nonperturbative parts represented by collinearly-divergent terms, which correspond to the matrix elements of a class of newly introduced generalized parton distributions (GPDs) [4, 5, 6]. These GPDs are hybrid objects, which combine properties of form factors and of ordinary parton distributions.

For WACS process, there is a general agreement that in asymptotic large momentum transfer region, the amplitude for Compton scattering is dominated by Brodsky-Lepage hard scattering picture [7, which is given by the convolution of a hard-scattering am-

plitude of collinear constituent partons and the distribution amplitude of hadrons. The perturbative contribution has been calculated in [8, 9, 10, to leading twist accuracy. However, the cross sections predicted in that approach are way below the existing Compton data, unless strongly asymmetric, i.e. end-point concentrated distribution amplitudes are used. This results in the conclusion that the cross sections are dominated by contributions from the soft end-point regions, where the assumptions of the leading twist perturbative calculation break down. Thus, the hard scattering model of Brodsky-Lepage, although likely to be the true asymptotic picture for exclusive reactions, does not seem to be dominant at moderately large momentum transfer, the kinematical region being accessible to present-day experiments.

Recently, a new mechanism has been introduced, in which the physics of handbag diagram is also of importance even for WACS reaction. As has been argued in [11, 12, 13, at moderately large momentum transfer, Compton scattering off protons approximately factorizes into a hard parton-photon subprocess and a soft-proton matrix element described by new form factors specific to Compton scattering. These new form factors represent moments of GPDs and can be modelled by overlaps of light-cone wave functions, which provide the link between exclusive and inclusive reactions. Using light-cone wave function overlaps as a model of the GPDs, detailed predictions of cross sections and polarization observables for real and virtual Compton scattering have been achieved in [12, 13. by using the leading order (LO) results for the hard parton-photon subprocess. 
In addition, the perturbative contributions appeared in the factorization formalisms for both DVCS and WACS processes have been calculated to one-loop level so far. The authors of [5, 14, 15, 16, have considered the one-loop corrections to DVCS process, while the next-to-leading order (NLO) QCD corrections to the parton level subprocess of real WACS processes have been calculated in [17. Some numerical predictions were also presented in the latter paper [17] based on the GPDs model proposed in [12.

The purpose of the present paper is to calculate the NLO corrections to the parton level virtual Compton scattering in wide-angle region. Our results together with the GPDs form factors will provide the necessary ingredients for calculating the wide-angle virtual Compton scattering at the NLO. These results may also be used to compare with other theoretical results in order to test the handbag mechanism and facilitate the interpretation of future experimental data that might be obtained at Jefferson Lab or at an ELFE-type accelerator at DESY or CERN.

The paper is organized as follows: Starting with the kinematical discussion, we calculate the LO amplitudes in Sect.2. Detailed calculations about the NLO results are given in Sect.3. Sect. 4 is devoted to the check of our calculations by comparing the divergent parts of our formulas with Catani's general formulas, and also by comparing the expressions of our formulas in the $-t \rightarrow 0$ (DVCS) and $Q^{2} \rightarrow 0$ (real WACS) limits with those results appeared in [5, 14, 15, 16, 17, 18. The paper terminates with a few concluding remarks (Sect. 5).

\section{LO amplitudes}

For the parton level virtual Compton process

$$
\gamma^{*}(q)+\operatorname{parton}(p) \longrightarrow \gamma\left(q^{\prime}\right)+\operatorname{parton}\left(p^{\prime}\right)
$$

we work in the center of mass system (CMS) of photon and parton. Neglecting the parton mass the different particle momenta can be chosen to be

$$
\begin{aligned}
q^{\mu} & =\left(q_{0}, 0,0,|\mathbf{p}|\right), \\
p^{\mu} & =|\mathbf{p}|(1,0,0,-1), \\
q^{\prime \mu} & =\left|\mathbf{p}^{\prime}\right|(1, \sin \theta, 0, \cos \theta), \\
p^{\prime \mu} & =\left|\mathbf{p}^{\prime}\right|(1,-\sin \theta, 0,-\cos \theta)
\end{aligned}
$$

in that frame. Here $\theta$ is the scattering angle of the outgoing real photon. $q_{0},|\mathbf{p}|,\left|\mathbf{p}^{\prime}\right|$ and $\theta$ are related to the Mandelstam variables $s=(p+q)^{2}, t=\left(p^{\prime}-p\right)^{2}$ and to the virtuality $\left(q^{2}=-Q^{2}\right)$ of the incoming photon by

$$
\begin{gathered}
q_{0}=\frac{s-Q^{2}}{2 \sqrt{s}}, \quad|\mathbf{p}|=\frac{s+Q^{2}}{2 \sqrt{s}} \\
\left|\mathbf{p}^{\prime}\right|=\frac{\sqrt{s}}{2}, \quad \cos \theta=1+\frac{2 t}{s+Q^{2}}
\end{gathered}
$$




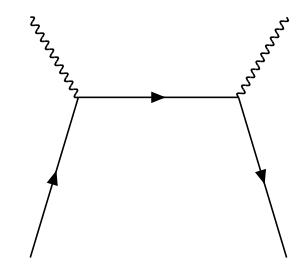

(a)

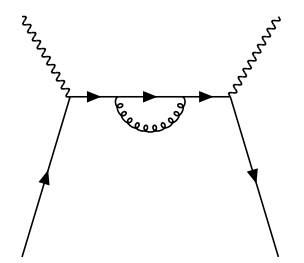

(b)

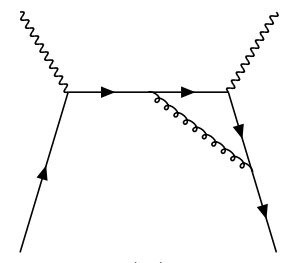

(d)

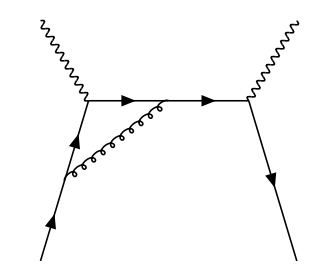

(c)

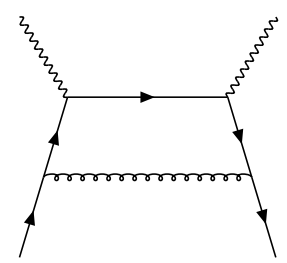

(e)

Figure 1: Feynman graphs for Compton scattering off on-shell quarks. a) is the LO graph, the others represent the NLO QCD corrections. Graphs with self-energy corrections to external fermions and those with interchanged interaction points of the photons are not shown.

In CMS, the polarization vectors of incoming virtual photon are

$$
\epsilon_{q}( \pm)=\mp \frac{1}{\sqrt{2}}(0,1, \pm i, 0), \quad \epsilon_{q}(0)=\frac{1}{Q}\left(|\mathbf{p}|, 0,0, q_{0}\right) .
$$

The polarization vector of the outgoing real photon is given by

$$
\epsilon_{q^{\prime}}\left(\mu^{\prime}\right)=\frac{1}{\sqrt{2}}\left(0,-\mu^{\prime} \cos \theta,-i, \mu^{\prime} \sin \theta\right)
$$

The tree amplitudes only receive contribution from scattering with quark. There are two Feynman diagrams which contribute to the LO amplitude for the reaction $\gamma^{*} q \rightarrow \gamma q$; a half of them is shown in Fig 1(a). The other half will be taken into account by using the crossing symmetry, i.e. the simultaneous replacement of $q \leftrightarrow-q^{\prime}$ and $\epsilon_{q} \leftrightarrow \epsilon_{q^{\prime}}^{*}$. The LO amplitude is

$$
\mathcal{M}_{\mu^{\prime} \lambda^{\prime}, \mu \lambda}^{(0)}=e_{q}^{2} e^{2} M_{\mu^{\prime} \lambda^{\prime}, \mu \lambda}^{(0)}
$$

where $e_{q}$ is the electric charge of quark $q$, and $\mu(\lambda)$ and $\mu^{\prime}\left(\lambda^{\prime}\right)$ denote the helicity of the initial and final state photons (partons), respectively. The amplitude $M_{\mu^{\prime} \lambda^{\prime}, \mu \lambda}^{(0)}$ is written 


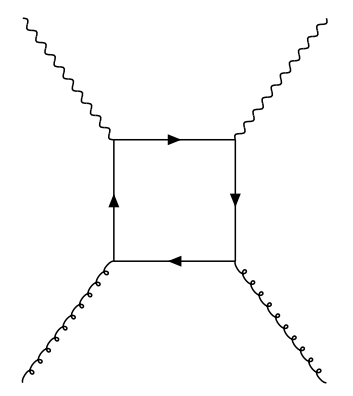

(a)

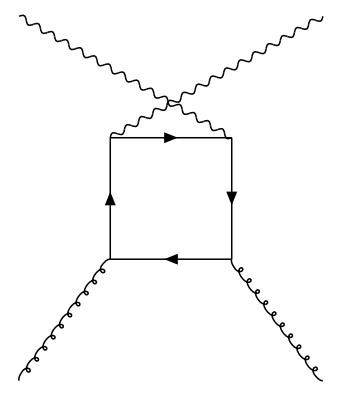

(b)

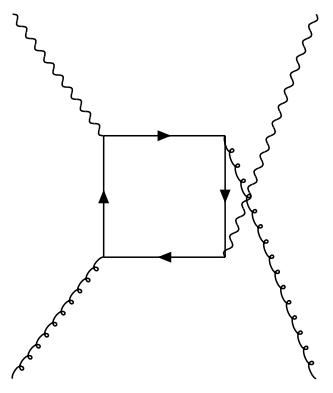

(c)

Figure 2: Sample Feynman graphs for photon-gluon scattering.

as

$$
M_{\mu^{\prime} \lambda^{\prime}, \mu \lambda}^{(0)}=\bar{u}\left(p^{\prime}, \lambda^{\prime}\right)\left[\oint_{q^{\prime}}^{*}\left(\mu^{\prime}\right) \frac{\not p+\not 1}{(p+q)^{2}+i \epsilon} \phi_{q}(\mu)+\oint_{q}(\mu) \frac{\not p-\not q^{\prime}}{\left(p-q^{\prime}\right)^{2}+i \epsilon} \phi_{q^{\prime}}^{*}\left(\mu^{\prime}\right)\right] u(p, \lambda) .
$$

Working out in the photon-parton CMS, we explicitly find

$$
\begin{aligned}
M_{++,++}^{(0)}=2 \sqrt{\frac{s}{-u}} \frac{s+Q^{2}}{s}, & M_{-+,-+}^{(0)}=2 \sqrt{\frac{-u}{s}} \frac{s}{s+Q^{2}}, \\
M_{-+,++}^{(0)}=2 \frac{Q^{2}}{s+Q^{2}} \frac{t}{\sqrt{-s u}}, & M_{++,-+}^{(0)}=0, \\
M_{-+, 0+}^{(0)}=2 \frac{Q}{s+Q^{2}} \sqrt{-2 t}, & M_{++, 0+}^{(0)}=0 .
\end{aligned}
$$

For the sake of legibility, explicit helicities are labeled only by their signs. Since the quarks are taken as massless, there is no quark helicity flip amplitude, i.e. $M_{\mu^{\prime}-\lambda, \mu \lambda}=0$, to any order of $\alpha_{s}$. Other helicity amplitudes can be obtained from those given in (8) by parity invariance:

$$
M_{-\mu^{\prime}-\lambda^{\prime},-\mu-\lambda}=(-1)^{\mu-\lambda-\mu^{\prime}+\lambda^{\prime}} M_{\mu^{\prime} \lambda^{\prime}, \mu \lambda}
$$

\section{One loop processes}

The NLO corrections to $\gamma^{*} q \rightarrow \gamma q$ can be calculated from the Feynman graphs (b)(e) depicted in Fig 1. We work in Feynman gauge and use dimensional regularization $(n=4-2 \epsilon)$. There are two mitigating factors which simplify the NLO corrections. The first is that the calculation does not contain ultraviolet (UV) singularities since the graphs in Fig. 1 do not contribute to the renormalization of the strong, electromagnetic, or weak coupling constants. The second is that the self-energy insertions on the external quark lines vanish due to the cancellation of the UV and infrared (IR) divergences [19]. Basically, what happens is that the UV and IR poles cancel when one does not distinguish between them. 
We evaluate these amplitudes using the Feynman parametrization technique. The loop integrals associated with the four-point function from the box diagrams shown in Fig П(e) are very difficult to evaluate when powers of the loop momenta appear in the numerator. However, one can express these tensor integrals in terms of lower rank tensor integrals with the same number of propagators and lower rank tensors with fewer propagators [20]. In the end, the four-point functions with powers of the loop momentum in the numerator are reduced to a four-point functions with a constant numerator and three- and two-point functions which are easier to evaluate. The all loop integrals can be reduced to a set of 13 scalar integrals which are given in Appendix.

The NLO corrections to the $\gamma^{*} q \rightarrow \gamma q$ amplitudes read

$$
\begin{aligned}
& M_{++,++}^{q(1)}=M_{++,++}^{(0)} \frac{\alpha_{s}}{\pi} C_{F} f(\epsilon)\left\{-\frac{1}{(-t)^{\epsilon}}\left(\frac{1}{2 \epsilon^{2}}+\frac{3}{4 \epsilon}\right)-\frac{9}{4}+\frac{\pi^{2}}{12}\right. \\
& +\frac{\left(2 s u-t Q^{2}\right)(s+u)+2(s+t) s u}{4(s+t)(s+u)(t+u)^{2}} t-\frac{\left(4 s t+s u+4 t^{2}+2 t u\right) s u}{4(s+t)^{2}(t+u)^{2}} \ln \frac{Q^{2}}{-u} \\
& \left.-\left(\frac{3}{4}+\frac{\left(2 Q^{2}+t\right) s t u}{2(s+u)^{2}(t+u)^{2}}\right) \ln \frac{-t}{Q^{2}}-\frac{f_{1}(s, t, u)}{4}-\frac{t^{2}}{4(t+u)^{2}} f_{2}(s, t, u)\right\}, \\
& M_{-+,-+}^{q(1)}=M_{-+,-+}^{(0)} \frac{\alpha_{s}}{\pi} C_{F} f(\epsilon)\left\{-\frac{1}{(-t)^{\epsilon}}\left(\frac{1}{2 \epsilon^{2}}+\frac{3}{4 \epsilon}\right)-\frac{9}{4}+\frac{\pi^{2}}{12}-\frac{t}{2(s+u)}\right. \\
& +\frac{2 t-u}{4 u}\left(\ln \frac{-t}{s}+i \pi\right)+\frac{\left(t u+s t-u Q^{2}\right) Q^{2}}{2(s+u)^{2} u} \ln \frac{-t}{Q^{2}} \\
& \left.-\frac{t^{2}}{4 u^{2}} f_{1}(s, t, u)-\frac{f_{2}(s, t, u)}{4}\right\} \text {, } \\
& M_{-+,++}^{q(1)}=M_{-+,++}^{(0)} \frac{\alpha_{s}}{\pi} C_{F} f(\epsilon)\left\{-\frac{1}{(-t)^{\epsilon}}\left(\frac{1}{2 \epsilon^{2}}+\frac{3}{4 \epsilon}\right)-\frac{9}{4}+\frac{\pi^{2}}{12}-\frac{3 s^{2}+4 s u+3 u^{2}}{4(s+u)^{2}} \ln \frac{-t}{Q^{2}}\right. \\
& \left.-\frac{f_{1}(s, t, u)+f_{2}(s, t, u)}{4}\right\}-\frac{\alpha_{s}}{2 \pi} C_{F} \frac{[(s+u)(t+u)+2 s u] t}{\sqrt{-s u}(s+u)\left(s+Q^{2}\right)}, \\
& M_{++,-+}^{q(1)}=\frac{\alpha_{s}}{\pi} C_{F} \sqrt{\frac{-u}{s}} \frac{t}{s+Q^{2}}\left\{\left(\frac{2 s+u}{(s+u)^{2}} \ln \frac{Q^{2}}{-t}+\frac{3 s+2 t}{2(s+t)^{2}} \ln \frac{Q^{2}}{-u}-\frac{f_{2}(s, t, u)}{2 s}\right) Q^{2}\right. \\
& \left.+\frac{s-u-2 Q^{2}}{2(s+t)(s+u)} s\right\} \text {, } \\
& M_{-+, 0+}^{q(1)}=M_{-+, 0+}^{(0)} \frac{\alpha_{s}}{\pi} C_{F} f(\epsilon)\left\{-\frac{1}{(-t)^{\epsilon}}\left(\frac{1}{2 \epsilon^{2}}+\frac{3}{4 \epsilon}\right)-\frac{9}{4}+\frac{\pi^{2}}{12}-\frac{1}{2}\left(\ln \frac{-t}{s}+i \pi\right)\right. \\
& \left.+\frac{u}{2(s+u)}-\frac{s^{2}-2 u t-u^{2}}{4(s+u)^{2}} \ln \frac{-t}{Q^{2}}-\frac{f_{2}(s, t, u)}{4}+\frac{t}{4 u} f_{1}(s, t, u)\right\}, \\
& M_{++, 0+}^{q(1)}=\frac{\alpha_{s}}{\pi} C_{F} \frac{Q \sqrt{-2 t}}{s+Q^{2}}\left\{\frac{s t-u^{2}-s u}{(s+u)^{2}} \ln \frac{-t}{Q^{2}}+\frac{2 s t-s u+2 t^{2}}{2(s+t)^{2}} \ln \frac{-u}{Q^{2}}\right. \\
& \left.+\frac{t}{2 s} f_{2}(s, t, u)+\frac{s t-2 s u-t u}{2(s+t)(s+u)}\right\} \text {, }
\end{aligned}
$$


where the functions $f(\epsilon)$ and $f_{i}(s, t, u), i=1,2,3$ are all defined in the Appendix. We see that the NLO amplitudes possess both non-zero imaginary parts and non-zero photon helicity flips.

At the one-loop level, there is a complication which we have to discuss next, namely gluons have to be considered as active partons as well. In contrast to the case of quarks, the partonic amplitudes now allow parton, i.e. gluon helicity flips to occur. The photongluon amplitudes can be calculated from the three graphs shown in Fig. 2, There are three further graphs contributing to order $\alpha_{s}$ which however reduce to the first three ones by reversing the fermion number flow. The fermion loop contributions satisfy the color decomposition

$$
\mathcal{M}_{a^{\prime} \mu^{\prime} \lambda^{\prime}, a \mu \lambda}^{g(1)}=\sum_{q} e_{q}^{2} e^{2} \delta_{a a^{\prime}} M_{\mu^{\prime} \lambda^{\prime}, \mu \lambda}^{g(1)}
$$

where $a_{1}$ and $a_{2}$ are color indices of incoming and outgoing gluons. After some algebra, we find for the gluon helicity non-flip amplitudes

$$
\begin{aligned}
& M_{++,++}^{g(1)}=\frac{\alpha_{s}}{\pi}\left\{-\frac{t^{2}+u^{2}}{2 s(t+u)} f_{2}(s, t, u)+\frac{\left(s t-2 s u-2 u^{2}\right) t}{(s+u)^{2}(t+u)} \ln \frac{Q^{2}}{-t}\right. \\
& \left.-\frac{\left(2 s t-s u+2 t^{2}\right) u}{(s+t)^{2}(t+u)} \ln \frac{Q^{2}}{-u}+\frac{\left(s-Q^{2}\right) t u}{(s+t)(s+u)(t+u)}\right\} \\
& M_{-+,-+}^{g(1)}=\frac{\alpha_{s}}{\pi}\left\{-\frac{\left(2 t^{2}+2 t u+u^{2}\right) s}{2 u^{2}(t+u)} f_{1}(s, t, u)+\frac{(2 t+u) s}{(t+u) u}\left(\ln \frac{-t}{s}+i \pi\right)\right. \\
& \left.-\frac{\left(2 s t+s u+3 t u+u^{2}\right) s Q^{2}}{(s+u)^{2}(t+u) u} \ln \frac{Q^{2}}{-t}-\frac{s t}{(s+u)(t+u)}\right\} \text {, } \\
& M_{-+,++}^{g(1)}=\frac{\alpha_{s}}{\pi}\left\{\frac{t Q^{2}}{2 u(t+u)} f_{1}(s, t, u)-\frac{t u Q^{2}}{(s+u)^{2}(t+u)} \ln \frac{Q^{2}}{-t}+\frac{s t}{(s+u)(t+u)}\right\} \text {, } \\
& M_{++,-+}^{g(1)}=\frac{\alpha_{s}}{\pi}\left\{\frac{u t Q^{2}}{s^{2}(t+u)} f_{2}(s, t, u)-\frac{(3 s+2 u) t u Q^{2}}{(s+u)^{2}(t+u) s} \ln \frac{Q^{2}}{-t}\right. \\
& \left.-\frac{(3 s+2 t) t u Q^{2}}{(s+t)^{2}(t+u) s} \ln \frac{Q^{2}}{-u}-\frac{\left(s-Q^{2}\right) t u}{(s+t)(t+u)(s+u)}\right\} \\
& M_{++, 0+}^{g(1)}=\frac{\alpha_{s}}{\pi}\left\{-\sqrt{\frac{u t}{2 s}} \frac{(t-u) Q}{s(t+u)} f_{2}(s, t, u)+\sqrt{\frac{2 u t}{s}} \frac{\left(2 s t-s u+t u-u^{2}\right) Q}{(s+u)^{2}(t+u)} \ln \frac{Q^{2}}{-t}\right. \\
& \left.+\sqrt{\frac{2 u t}{s}} \frac{\left(s t-2 s u+t^{2}-t u\right) Q}{(s+t)^{2}(t+u)} \ln \frac{Q^{2}}{-u}-\frac{\sqrt{2 u t s}(t-u) Q}{(s+t)(s+u)(t+u)}\right\}, \\
& M_{-+, 0+}^{g(1)}=\frac{\alpha_{s}}{\pi}\left\{\sqrt{\frac{u t s}{2}} \frac{t Q}{u^{2}(t+u)} f_{1}(s, t, u)-\frac{\sqrt{2 u t s} Q}{(t+u) u}\left(\ln \frac{-t}{s}+i \pi\right)\right. \\
& \left.+\frac{\sqrt{2 u t s}(s+2 u) Q^{3}}{(s+u)^{2}(t+u) u} \ln \frac{Q^{2}}{-t}+\frac{\sqrt{2 u t s} Q}{(s+u)(t+u)}\right\} \text {, }
\end{aligned}
$$


and for the gluon helicity flip amplitudes

$$
\begin{aligned}
M_{++,+-}^{g(1)}= & \frac{\alpha_{s}}{\pi}, \\
M_{-+,--}^{g(1)}= & \frac{\alpha_{s}}{\pi}\left(\frac{u Q^{2}}{2 t(t+u)} f_{3}(s, t, u)+\frac{s u}{(s+t)(t+u)}-\frac{t u Q^{2}}{(s+t)^{2}(t+u)} \ln \frac{Q^{2}}{-u}\right) \\
M_{-+,+-}^{g(1)}= & \frac{\alpha_{s}}{\pi}\left(-\frac{\left(t^{2}+2 t u+2 u^{2}\right) s}{2 t^{2}(t+u)} f_{3}(s, t, u)-\frac{s u}{(s+t)(t+u)}\right. \\
& \left.+\frac{s(t+2 u)}{(t+u) t}\left(\ln \frac{Q^{2}}{s}+i \pi\right)+\frac{s u\left(2 s t+2 s u+2 t^{2}+3 t u\right)}{(s+t)^{2}(t+u) t} \ln \frac{Q^{2}}{-u}\right) \\
M_{++,--}^{g(1)}= & \frac{\alpha_{s}}{\pi}, \\
M_{++, 0-}^{g(1)}= & 0, \\
M_{-+, 0-}^{g(1)}= & \frac{\alpha_{s}}{\pi}\left(\sqrt{\frac{u t s}{2}} \frac{u Q}{t^{2}(t+u)} f_{3}(s, t, u)+\frac{\sqrt{2 u t s} Q}{(s+t)(t+u)}\right. \\
& \left.-\frac{\sqrt{2 u t s} Q}{(t+u) t}\left(\ln \frac{Q^{2}}{s}+i \pi\right)-\frac{\sqrt{2 u t s} Q\left(s t+s u+t^{2}+2 t u\right)}{(s+t)^{2}(t+u) t} \ln \frac{Q^{2}}{-u}\right) .
\end{aligned}
$$

We find that for all helicity non-flip amplitudes, the scaling behavior of $-t \rightarrow 0$ are satisfied, which is demanded by the angular momentum conservation.

\section{Comparison with other results}

We find that those helicity amplitudes which are non-zero at LO, turn out to include infrared divergent parts which go as an universal form

$$
-M_{\mu^{\prime} \lambda^{\prime}, \mu \lambda}^{(0)} \frac{\alpha_{s}}{2 \pi} C_{F} f(\epsilon)\left(\frac{1}{\epsilon^{2}}+\frac{3}{2 \epsilon}\right) \frac{1}{(-t)^{\epsilon}},
$$

where the $1 / \epsilon^{2}$ term appears as a consequence of overlapping soft and collinear divergences. Catani presented a general formula for the structure of infrared divergences of any QCD amplitude [21]. For the case of the one-loop amplitude, Catani's formula is

$$
M^{(0)} \frac{1}{2} \frac{-e^{\epsilon \psi(1)}}{\Gamma(1-\epsilon)} \sum_{i} \frac{1}{\mathbf{T}_{i}{ }^{2}}\left(\mathbf{T}_{i}{ }^{2} \frac{1}{\epsilon^{2}}+\gamma_{i} \frac{1}{\epsilon}\right) \sum_{j \neq i} \mathbf{T}_{i} \cdot \mathbf{T}_{j}\left(\frac{\mu^{2} e^{-i \lambda_{i j} \pi}}{2 p_{i} \cdot p_{j}}\right)^{\epsilon} \frac{\alpha_{s} S_{\epsilon}}{2 \pi},
$$

where $S_{\epsilon}$ is the typical phase-space volume factor in $d=4-2 \epsilon\left(\gamma_{E}=-\psi(1)=0.5772 \cdots\right.$ is the Euler number)

$$
S_{\epsilon}=\exp [\epsilon(\ln 4 \pi+\psi(1))]
$$

In (30), the sum is over all external partons of the amplitudes. $\lambda_{i j}=+1$ if $i$ and $j$ are both incoming or outgoing partons and $\lambda_{i j}=0$ otherwise. The color charge $\mathbf{T}_{i}=\left\{T_{i}^{a}\right\}$ 
is a vector with respect to the color indices $a$ of the emitted gluon, and an $S U(N)$ matrix with respect to the color indices of the parton $i$. Applying (30) to the process we consider here, one may convert two of the partons to photons by setting $\mathbf{T}_{i} \cdot \mathbf{T}_{\gamma} \rightarrow 0$ and $\mathbf{T}_{i} \cdot \mathbf{T}_{j}=-\mathbf{T}_{i}^{2}$ to obtain the simplified formula

$$
-M^{(0)} \frac{\alpha_{s}}{2 \pi} \frac{\exp (\epsilon \ln 4 \pi)}{\Gamma(1-\epsilon)} C_{F}\left(\frac{1}{\epsilon^{2}}+\frac{3}{2 \epsilon}\right)\left(\frac{\mu^{2}}{-t}\right)^{\epsilon},
$$

the divergent part of which is exactly equal to the one given in eq.(29). This agreement provides a stringent check of our amplitudes.

To perform further checks on the reliability of amplitudes, we compare the amplitudes of real photon-parton scattering processes obtained from our formulas with those already existed results. The helicity amplitudes with one-loop corrections to real Compton process $\gamma q \rightarrow \gamma q$ can be derived from (10)-(15) by taking $Q^{2}=0$, and they are

$$
\begin{aligned}
M_{+,++}^{q(1)} & =f(\epsilon) \frac{C_{F} \alpha_{s}}{\pi}\left\{-\frac{1}{(-t) \epsilon}\left(\frac{1}{\epsilon^{2}}+\frac{3}{2 \epsilon}\right)-\frac{7}{2}+\frac{\pi^{2}}{6}\right. \\
& \left.+\frac{2 t-s}{2 s} \ln \frac{t}{u}+\frac{1}{2} \ln ^{2} \frac{-t}{s}+\frac{t^{2}}{2 s^{2}}\left(\ln ^{2} \frac{t}{u}+\pi^{2}\right)+i \pi \ln \frac{-t}{s}\right\} \sqrt{\frac{s}{-u}}, \\
M_{+,--}^{q(1)} & =f(\epsilon) \frac{C_{F} \alpha_{s}}{\pi}\left\{-\frac{1}{(-t)^{\epsilon}}\left(\frac{1}{\epsilon^{2}}+\frac{3}{2 \epsilon}\right)-\frac{7}{2}+\frac{\pi^{2}}{6}\right. \\
& +\frac{2 t-u}{2 u} \ln \frac{-t}{s}+\frac{1}{2}\left(\ln ^{2} \frac{t}{u}+\pi^{2}\right)+\frac{t^{2}}{2 u^{2}} \ln ^{2} \frac{-t}{s} \\
M_{+,+-}^{q(1)} & =-\frac{C_{F} \alpha_{s}}{2 \pi}\left(\sqrt{\frac{s}{-u}}+\sqrt{\frac{-u}{s}}\right), \\
M_{+,-+}^{(1)} & \left.\left.=-\frac{C_{F}^{2} \alpha_{s}}{2 \pi}\left(\sqrt{\frac{-t}{s}}\right)\right\} \sqrt{\frac{-u}{s}}, \sqrt{\frac{-u}{s}}\right), \\
M_{+, 0+}^{q(1)} & =0 \\
M_{+, 0-}^{q(1)} & =0 .
\end{aligned}
$$

We can see that the contributions from longitudinal photon disappear and all other amplitudes agree with those given in [17]. Applying $Q^{2}=0$ to the real photon-gluon scattering process $\gamma g \rightarrow \gamma g$, (17)-(28) can be simplified as

$$
\begin{aligned}
& M_{++,++}^{g(1)}=-\frac{\alpha_{s}}{\pi}\left\{\frac{t^{2}+u^{2}}{2 s^{2}}\left(\ln ^{2} \frac{t}{u}+\pi^{2}\right)+\frac{s+2 t}{s} \ln \frac{t}{u}+1\right\} \\
& M_{++,--}^{g(1)}=-\frac{\alpha_{s}}{\pi}\left\{\frac{s^{2}+t^{2}}{2 u^{2}} \ln ^{2} \frac{-t}{s}+\frac{t-s}{u} \ln \frac{-t}{s}+1+i \pi\left(\frac{t-s}{u}+\frac{s^{2}+t^{2}}{u^{2}} \ln \frac{-t}{s}\right)\right\}
\end{aligned}
$$




$$
\begin{aligned}
& M_{++,+-}^{g(1)}=\frac{\alpha_{s}}{\pi}, \\
& M_{++,-+}^{g(1)}=\frac{\alpha_{s}}{\pi}, \\
& M_{++, 0+}^{g(1)}=0 \\
& M_{++, 0-}^{g(1)}=0 \\
& M_{+-,++}^{g(1)}=\frac{\alpha_{s}}{\pi}, \\
& M_{+-,--}^{g(1)}=\frac{\alpha_{s}}{\pi}, \\
& M_{+-,+-}^{g(1)}=-\frac{\alpha_{s}}{\pi}\left\{\frac{s^{2}+u^{2}}{2 t^{2}} \ln ^{2} \frac{-u}{s}+\frac{u-s}{t} \ln \frac{-u}{s}+1\right. \\
& M_{+-,-+}^{g(1)}=\frac{\alpha_{s}}{\pi}, \\
& M_{+-, 0+}^{g(1)}=0, \\
& M_{+-, 0-}^{g(1)}=0,
\end{aligned}
$$

which are also equal to the results obtained in [17] and [18.

To compare with the DVCS results, let us take the forward limit, i.e. $-t \rightarrow 0$. Then, from (8), we can see that most of the LO helicity amplitudes vanish except

$$
M_{++,++}^{(0)}=2 \sqrt{\frac{s+Q^{2}}{s}}, \quad M_{-+,-+}^{(0)}=2 \sqrt{\frac{s}{s+Q^{2}}} .
$$

Since the scalar integrals (58), (59), (60) and (70) in Appendix are not well-defined at the point $-t=0$, our methods described above can not be directly applied to the case of DVCS. However, this difficulty may be avoided by taking the trace over the numerator of box integrals. In the DVCS limit $(-t=0)$, from (2) we have $p^{\prime}=\frac{s}{s+Q^{2}} p$ and the product of two Dirac spinors can be written as $u_{\alpha}\left(p^{\prime}, \lambda\right) \bar{u}_{\beta}(p, \lambda) \sim\left(1+\lambda \gamma_{5}\right) p$, therefore the numerators of the box integrals is of the trace form. The trace of the numerator can be expressed as a sum of terms which cancel one of the propagators in the denominator. This can be done because $k^{2}$ and $k \cdot p$ can be written as linear combinations of $k^{2}$ and $(k+p)^{2}$, where $k$ is the loop momentum, and the trace only includes these two type of terms. Since we have shown that the numerator will be linear combination of two different denominators, the four-propagator integral will in general become two three-propagator integrals. After this procedure, we do not need to carry out the calculation of the four questionable scalar integrals mentioned above to obtain the DVCS amplitudes, and the calculation becomes much simpler. After some algebras, we find the NLO results for the DVCS helicity amplitudes

$$
M_{++,++}^{(1)}=M_{++,++}^{(0)}\left(-\frac{\alpha_{s} C_{F} f(\epsilon)}{4 \pi}\right)\left\{\frac{1}{\left(Q^{2}\right) \epsilon} \frac{1}{\epsilon}\left[3+2\left(\ln \frac{s}{Q^{2}}-i \pi\right)\right]\right.
$$




$$
\begin{aligned}
& \left.+9-\ln \frac{-u}{Q^{2}}-\frac{2 s}{s+Q^{2}}\left(\ln \frac{s}{Q^{2}}-i \pi\right)-\left(\ln \frac{s}{Q^{2}}-i \pi\right)^{2}\right\}, \\
M_{-+,-+}^{(1)}= & M_{-+,-+}^{(0)}\left(-\frac{\alpha_{s} C_{F} f(\epsilon)}{4 \pi}\right)\left\{\frac{1}{\left(Q^{2}\right)^{\epsilon}} \frac{1}{\epsilon}\left(3+2 \ln \frac{-u}{Q^{2}}\right)\right. \\
& \left.+9-\left(\ln \frac{s}{Q^{2}}-i \pi\right)-\frac{2\left(s+Q^{2}\right)}{s} \ln \frac{-u}{Q^{2}}-\ln ^{2} \frac{-u}{Q^{2}}\right\} .
\end{aligned}
$$

The $1 / \epsilon^{2}$ divergent terms vanish and only $1 / \epsilon$ divergence is left, this is due to the fact that there is no soft divergence in the $-t=0$ limit. Except for an overall normalization factor, the symmetric and antisymmetric quark amplitudes defined in [5] can be written as

$$
T_{q}^{(i j)}=M_{i-, i-}+M_{j+, j+}, \quad T_{q}^{[i j]}=M_{i-, i-}-M_{j+, j+} .
$$

The CM frame chosen in this paper is different from the CM frame chosen in [5], and the relation between the kinematic variables used in these two papers can be expressed as

$$
s=\frac{1-x_{B}}{x_{B}} \tilde{Q}^{2}, \quad Q^{2}=2 \tilde{Q}^{2}
$$

where $\tilde{Q}^{2}$ represents the variable $Q^{2}$ defined in [5]. After the substitutions of (55), the two amplitudes derived from (54) are exactly equal to the corresponding equations (24) and (25) in [5] when the limits of $-t \rightarrow 0$ and $M^{2} \rightarrow 0$ are taken for the later case. By checking in the similar way, our results in DVCS limit are also shown to be in agreement with other existing one-loop results in [14, 15, 16].

\section{Conclusion and discussion}

As a complement to the results given in [13], we have calculated the NLO QCD corrections to the subprocess helicity amplitudes for virtual Compton scattering. The divergent parts, being consistent with Catani's general formulas, exhibit the same behavior as those in electromagnetic form factor and therefore can be attributed to the form factors introduced in [12] which are specific to Compton scattering. Our finite results can be used to the predictions for various virtual Compton observables and are compared to the leading contribution given in [13]. One of the interesting physical quantities is the beam asymmetry for $e p \rightarrow e p \gamma$

$$
A_{L}=\frac{d \sigma(+)-d \sigma(-)}{d \sigma(+)+d \sigma(-)}
$$

where the labels + and - denote the lepton beam helicity. This quantity depends on the relative phase between the complex virtual Compton amplitudes and the real BetheHeitler ones. In the LO soft physics approach, $A_{L}$ is zero because all amplitudes are real. 
However, since the $\alpha_{s}$ corrections in the photon-parton subprocess include imaginary parts, $A_{L}$ may become non-zero in the NLO soft physics approach [22. This must be a good test for the handbag mechanism by comparing with the data which will be obtained in the forthcoming experiments at Jefferson Lab or ELFE-type accelerators.

\section{Acknowledgments}

This work started when one of the authors(H.W.H.) was at Kobe university as the postdoctoral research fellow (No. P99221) of the Japan Society of the Promotion of Science (JSPS) and H.W.H. would like to thank the Monbusho's Grand-in-Aid for the JSPS postdoctoral fellow for financial support. The authors also acknowledge useful comments from Dieter Müller.

\section{References}

[1] F. M. Dittes, D. Müller, D. Robaschik, B. Geyer and J. Horejsi, Phys. Lett. B 209, 325 (1988).

[2] D. Müller, D. Robaschik, B. Geyer, F. M. Dittes and J. Horejsi, Fortsch. Phys. 42, 101 (1994) arXiv:hep-ph/9812448.

[3] X. Ji, Phys. Rev. Lett. 78, 610 (1997); Phys. Rev. D55, 7114 (1997)

[4] A. V. Radyushkin, Phys. Rev. D 56, 5524 (1997) arXiv:hep-ph/9704207.

[5] X. D. Ji and J. Osborne, Phys. Rev. D58, 094018 (1998) arXiv:hep-ph/9801260.

[6] J. C. Collins and A. Freund, Phys. Rev. D59, 074009 (1999) arXiv:hep-ph/9801262.

[7] G.P. Lepage and S.J. Brodsky, Phys. Rev. D22, 2157 (1980).

[8] G. R. Farrar and H. Zhang, Phys. Rev. D41, 3348 (1990) [Erratum-ibid. D42, 3348 (1990)].

[9] A. Kronfeld and B. Nižić, Phys. Rev. D44, 3445 (1991); Erratum Phys. Rev. D46, 2272 (1992).

[10] M. Vanderhaeghen, P.A.M. Guichon and J. Van de Wiele, Nucl. Phys. A622, 144c (1997).

[11] A. V. Radyushkin, Phys. Rev. D58, 114008 (1998) arXiv:hep-ph/9803316.

[12] M. Diehl, Th. Feldmann, R. Jakob and P. Kroll, Eur. Phys. J. C8, 409 (1999) arXiv:hep-ph/9811253. 
[13] M. Diehl, T. Feldmann, R. Jakob and P. Kroll, Phys. Lett. B460, 204 (1999) arXiv:hep-ph/9903268.

[14] A. V. Belitsky and D. Müller, Phys. Lett. B 417, 129 (1998) arXiv:hep-ph/9709379.

[15] L. Mankiewicz, G. Piller, E. Stein, M. Vanttinen and T. Weigl, Phys. Lett. B 425, 186 (1998) arXiv:hep-ph/9712251.

[16] A. V. Belitsky and A. Schäfer, Nucl. Phys. B 527, 235 (1998) arXiv:hep-ph/9801252.

[17] H. W. Huang, P. Kroll, T. Morii, Eur. Phys. J. C23, 301 (2002) arXiv:hep-ph/0110208.

[18] Z. Bern, A. De Freitas, L. Dixon, JHEP 0109,037 (2001) arXiv:hep-ph/0109078.

[19] W. L. Van Neerven, Nucl. Phys. B268, 453 (1986).

[20] G. Passarino, M. Veltman, Nucl. Phys. B160, 151 (1979).

[21] S. Catani, Phys. Lett. B427, 161 (1998) arXiv:hep-ph/9802439.

[22] H. W. Huang and T. Morii, in preparation. 


\section{A Scalar loop integrals}

The loop integrals from the one-loop graphs of Fig. 1 and Fig. 2 can be reduced to a set of 13 scalar integrals which are given in this Appendix. They all include a common overall factor

$$
f(\epsilon)=\frac{i \Gamma(1+\epsilon)\left(4 \pi \mu^{2}\right)^{\epsilon}}{16 \pi^{2}}
$$

Among these 13 integrals, the four-point integrals $D_{0}^{i}(i=1-3)$ and the three-point integrals $C_{0}^{i}(i=1-6)$ are infrared divergent and ultraviolet finite, while the twopoint integrals $B_{0}^{i}(i=1-4)$ include only UV divergence. However, the UV divergent term containing $\epsilon$ cancels when the two-point functions are combined to form the tensor integrals and the UV divergences of the individual graphs also cancel in the sum and, therefore, the final amplitudes are UV safe.

The four-point functions that we need can be expressed as

$$
\begin{aligned}
D_{0}^{1} & =\mu^{2 \epsilon} \int \frac{d^{n} k}{(2 \pi)^{n}} \frac{1}{\left(k^{2}+i \varepsilon\right)\left[(p+k)^{2}+i \varepsilon\right]\left[(p+q+k)^{2}+i \varepsilon\right]\left[\left(p^{\prime}+k\right)^{2}+i \varepsilon\right]} \\
& =\frac{f(\epsilon)}{s t}\left\{\frac{2}{\epsilon^{2}}\left(\frac{1}{(-s-i \varepsilon)^{\epsilon}}+\frac{1}{(-t)^{\epsilon}}-\frac{1}{\left(Q^{2}\right)^{\epsilon}}\right)-\frac{\pi^{2}}{3}+f_{1}(s, t, u)\right\}, \\
D_{0}^{2} & =\mu^{2 \epsilon} \int \frac{d^{n} k}{(\pi)^{n}} \frac{1}{\left(k^{2}+i \varepsilon\right)\left[(p+k)^{2}+i \varepsilon\right]\left[\left(p-q^{\prime}+k\right)^{2}+i \varepsilon\right]\left[\left(p^{\prime}+k\right)^{2}+i \varepsilon\right]} \\
& =\frac{f(\epsilon)}{u t}\left\{\frac{2}{\epsilon^{2}}\left(\frac{1}{(-u)^{\epsilon}}+\frac{1}{(-t)^{\epsilon}}-\frac{1}{\left(Q^{2}\right)^{\epsilon}}\right)-\frac{\pi^{2}}{3}+f_{2}(s, t, u)\right\}, \\
D_{0}^{3} & =\mu^{2 \epsilon} \int \frac{d^{n} k}{(2 \pi)^{n}} \frac{1}{\left(k^{2}+i \varepsilon\right)\left[(p+k)^{2}+i \varepsilon\right]\left[(p+q+k)^{2}+i \varepsilon\right]\left[\left(q^{\prime}+k\right)^{2}+i \varepsilon\right]} \\
& =\frac{f(\epsilon)}{s u}\left\{\frac{2}{\epsilon^{2}}\left(\frac{1}{(-s-i \varepsilon)^{\epsilon}}+\frac{1}{(-u)^{\epsilon}}-\frac{1}{\left(Q^{2}\right)^{\epsilon}}\right)-\frac{\pi^{2}}{3}+f_{3}(s, t, u)\right\} .
\end{aligned}
$$

where

$$
\begin{aligned}
f_{1}(s, t, u)= & \ln ^{2} \frac{-t}{s+Q^{2}}+\ln \frac{-t}{s+Q^{2}} \ln \frac{-u}{s}-2 \ln \frac{-t}{s+Q^{2}} \ln \frac{-t}{s}+\operatorname{Li}_{2}\left(\frac{-u}{s+Q^{2}}\right) \\
& -\operatorname{Li}_{2}\left(1+\frac{Q^{2}}{t}\right)-\operatorname{Li}_{2}\left(1-\frac{Q^{2} u}{s t}\right)+\operatorname{Li}_{2}\left(1+\frac{u}{s}\right)+\operatorname{Li}_{2}\left(\frac{s}{s+Q^{2}}\right) \\
& -2 i \pi \ln \frac{-t}{s+Q^{2}}, \\
f_{3}(s, t, u)= & \ln ^{2} \frac{-u}{s+Q^{2}}+\ln \frac{-u}{s+Q^{2}} \ln \frac{-t}{s}-2 \ln \frac{-u}{s+Q^{2}} \ln \frac{-u}{s}+\operatorname{Li}_{2}\left(\frac{-t}{s+Q^{2}}\right) \\
& -\operatorname{Li}_{2}\left(1+\frac{Q^{2}}{u}\right)-\operatorname{Li}_{2}\left(1-\frac{Q^{2} t}{s u}\right)+\operatorname{Li}_{2}\left(1+\frac{t}{s}\right)+\operatorname{Li}_{2}\left(\frac{s}{s+Q^{2}}\right) \\
& -2 i \pi \ln \frac{-u}{s+Q^{2}} .
\end{aligned}
$$


For $Q^{2}>-u$,

$$
\begin{aligned}
f_{2}(s, t, u)= & \ln ^{2}\left(\frac{-t}{u+Q^{2}}\right)+\ln \left(\frac{-t}{u+Q^{2}}\right) \ln \left(\frac{s}{-u}\right)-2 \ln \left(\frac{-t}{u+Q^{2}}\right) \ln \frac{t}{u} \\
& +\operatorname{Li}_{2}\left(\frac{-s}{u+Q^{2}}\right)-\operatorname{Li}_{2}\left(1+\frac{Q^{2}}{t}\right)-\operatorname{Li}_{2}\left(1-\frac{Q^{2} s}{t u}\right)+\operatorname{Li}_{2}\left(1+\frac{s}{u}\right) \\
& +\operatorname{Li}_{2}\left(\frac{u}{u+Q^{2}}\right),
\end{aligned}
$$

while for $Q^{2}<-u$,

$$
\begin{aligned}
f_{2}(s, t, u)= & \ln ^{2} \frac{t}{u+Q^{2}}+\ln \frac{t}{u+Q^{2}} \ln \frac{s}{-u}-2 \ln \frac{t}{u+Q^{2}} \ln \frac{t}{u}-\frac{\pi^{2}}{3}-\frac{1}{2} \ln ^{2} \frac{-s}{u+Q^{2}} \\
& -\frac{1}{2} \ln ^{2} \frac{u}{u+Q^{2}}-\operatorname{Li}_{2}\left(\frac{u+Q^{2}}{-s}\right)-\operatorname{Li}_{2}\left(1-\frac{Q^{2} s}{t u}\right)+\operatorname{Li}_{2}\left(1+\frac{s}{u}\right) \\
& -\operatorname{Li}_{2}\left(\frac{u+Q^{2}}{u}\right)-\operatorname{Li}_{2}\left(1+\frac{Q^{2}}{t}\right) .
\end{aligned}
$$

In the real photon case $\left(Q^{2} \rightarrow 0\right)$, we have the simplified results

$$
\begin{aligned}
& f_{1}(s, t, u)=-\ln ^{2} \frac{-t}{s}-2 i \pi \ln \frac{-t}{s}, \\
& f_{3}(s, t, u)=-\ln ^{2} \frac{-u}{s}-2 i \pi \ln \frac{-u}{s}, \\
& f_{2}(s, t, u)=-\ln ^{2} \frac{t}{u}-\pi^{2} .
\end{aligned}
$$

The three-point functions are

$$
\begin{aligned}
C_{0}^{1} & =\mu^{2 \epsilon} \int \frac{d^{n} k}{(2 \pi)^{n}} \frac{1}{\left(k^{2}+i \varepsilon\right)\left[(p+k)^{2}+i \varepsilon\right]\left[(p+q+k)^{2}+i \varepsilon\right]} \\
& =\frac{f(\epsilon)}{\left(s+Q^{2}\right)}\left\{\frac{1}{(-s-i \varepsilon)^{\epsilon}}-\frac{1}{\left(Q^{2}\right)^{\epsilon}}\right\} \frac{1}{\epsilon^{2}}, \\
C_{0}^{2} & =\mu^{2 \epsilon} \int \frac{d^{n} k}{(2 \pi)^{n}} \frac{1}{\left(k^{2}+i \varepsilon\right)\left[\left(p^{\prime}+k\right)^{2}+i \varepsilon\right]\left[(p+q+k)^{2}+i \varepsilon\right]} \\
& =\frac{f(\epsilon)}{s}\left\{\frac{1}{\epsilon^{2}} \frac{1}{(-s-i \varepsilon)^{\epsilon}}-\frac{\pi^{2}}{6}\right\}, \\
C_{0}^{3} & =\mu^{2 \epsilon} \int \frac{d^{n} k}{(2 \pi)^{n}} \frac{1}{\left(k^{2}+i \varepsilon\right)\left[(p+k)^{2}+i \varepsilon\right]\left[\left(p^{\prime}+k\right)^{2}+i \varepsilon\right]} \\
& =\frac{f(\epsilon)}{t}\left\{\frac{1}{\epsilon^{2}} \frac{1}{(-t)^{\epsilon}}-\frac{\pi^{2}}{6}\right\}, \\
C_{0}^{4} & =\mu^{2 \epsilon} \int \frac{d^{n} k}{(2 \pi)^{n}} \frac{1}{\left(k^{2}+i \varepsilon\right)\left[(k-q)^{2}+i \varepsilon\right]\left[\left(k-q^{\prime}\right)^{2}+i \varepsilon\right]}
\end{aligned}
$$




$$
\begin{aligned}
& =\frac{f(\epsilon)}{\left(t+Q^{2}\right)}\left\{\frac{1}{(-t)^{\epsilon}}-\frac{1}{\left(Q^{2}\right)^{\epsilon}}\right\} \frac{1}{\epsilon^{2}}, \\
C_{0}^{5} & =\mu^{2 \epsilon} \int \frac{d^{n} k}{(2 \pi)^{n}} \frac{1}{\left(k^{2}+i \varepsilon\right)\left[(p+k)^{2}+i \varepsilon\right]\left[\left(p-q^{\prime}+k\right)^{2}+i \varepsilon\right]} \\
& =\frac{f(\epsilon)}{u}\left\{\frac{1}{\epsilon^{2}} \frac{1}{(-u)^{\epsilon}}-\frac{\pi^{2}}{6}\right\}, \\
C_{0}^{6} & =\mu^{2 \epsilon} \int \frac{d^{n} k}{(2 \pi)^{n}} \frac{1}{\left(k^{2}+i \varepsilon\right)\left[\left(p^{\prime}+k\right)^{2}+i \varepsilon\right]\left[\left(p^{\prime}-q+k\right)^{2}+i \varepsilon\right]} \\
& =\frac{f(\epsilon)}{\left(u+Q^{2}\right)}\left\{\frac{1}{(-u)^{\epsilon}}-\frac{1}{\left(Q^{2}\right)^{\epsilon}}\right\} \frac{1}{\epsilon^{2}} .
\end{aligned}
$$

The two-point functions are

$$
\begin{aligned}
B_{0}^{1} & =\mu^{2 \epsilon} \int \frac{d^{n} k}{(2 \pi)^{n}} \frac{1}{\left(k^{2}+i \varepsilon\right)\left[(p+q+k)^{2}+i \varepsilon\right]} \\
& =f(\epsilon)\left(\frac{1}{\epsilon} \frac{1}{(-s-i \varepsilon)^{\epsilon}}+2\right), \\
B_{0}^{2} & =\mu^{2 \epsilon} \int \frac{d^{n} k}{(2 \pi)^{n}} \frac{1}{\left(k^{2}+i \varepsilon\right)\left[\left(p-p^{\prime}+k\right)^{2}+i \varepsilon\right]} \\
& =f(\epsilon)\left(\frac{1}{\epsilon} \frac{1}{(-t)^{\epsilon}}+2\right), \\
B_{0}^{3} & =\mu^{2 \epsilon} \int \frac{d^{n} k}{(2 \pi)^{n}} \frac{1}{\left(k^{2}+i \varepsilon\right)\left[(q+k)^{2}+i \varepsilon\right]} \\
& =f(\epsilon)\left(\frac{1}{\epsilon} \frac{1}{\left(Q^{2}\right)^{\epsilon}}+2\right), \\
B_{0}^{4} & =\mu^{2 \epsilon} \int \frac{d^{n} k}{(2 \pi)^{n}} \frac{1}{\left(k^{2}+i \varepsilon\right)\left[\left(p-q^{\prime}+k\right)^{2}+i \varepsilon\right]} \\
& =f(\epsilon)\left(\frac{1}{\epsilon} \frac{1}{(-u)^{\epsilon}}+2\right) .
\end{aligned}
$$

